\title{
Helmholtz bright and black soliton splitting at nonlinear interfaces
}

\author{
Julio Sánchez-Curto* and Pedro Chamorro-Posada \\ Departamento de Teoría de la Señal y Comunicaciones e Ingeniería Telemática, Universidad de Valladolid, \\ ETSI Telecomunicación, Paseo Belén 15, E-47011 Valladolid, Spain
}

\author{
Graham S. McDonald \\ Joule Physics Laboratory, Materials and Physics Research Centre, School of Computing, Science and Engineering, \\ University of Salford, Salford M5 4WT, United Kingdom \\ (Received 14 November 2011; published 24 January 2012)
}

\begin{abstract}
Soliton breakup occurring at the planar boundary separating two Kerr focusing and defocusing media is analyzed within the framework of the Helmholtz theory where the full angular content of the problem is preserved. We show that the number of solitons resulting from bright soliton breakup depends on the soliton angle of incidence, contrary to the predictions of previous paraxial analyses. The generation of multiple gray solitons resulting from the interaction of a dark soliton at Kerr defocusing interfaces is studied and the main properties of the output dark soliton pattern are revealed.
\end{abstract}

DOI: 10.1103/PhysRevA.85.013836

PACS number(s): 42.65.Tg, 42.25.Gy

\section{INTRODUCTION}

The planar boundary separating two dielectric media, where at least one of them is nonlinear, can produce the breakup of an incident soliton beam into multiple output solitons. This effect was numerically observed in the seminal works on nonlinear interfaces [1,2] for Gaussian beams at the interface separating linear and nonlinear (Kerr-type) media. Multilayered structures such as nonlinear waveguides, widely studied during the 1980s, were also found to emit multisoliton patterns in the nonlinear substrate $[3,4]$ from a linear guiding film covered by a linear cladding. These works focused on linear-nonlinear configurations; in contrast, a planar boundary separating Kerr-type and linear media has been recently proposed to induce fission of vector solitons [5] or multisoliton bound states [6] in the medium where the solitons initially propagate. In this scenario, the number of solitons resulting from the reflection of multisoliton bound states has been reported to possess a strong angular character [6].

The physics of solitons impinging on planar boundaries separating two Kerr-type media have been studied using the nonlinear Schrödinger (NLS) equation where the paraxial approximation is assumed [7,8]. The particlelike approach [8] has succeeded in explaining a large variety of phenomena arising at nonlinear interfaces. The limitations of such a model to address the breakup of a particle into different subparticles as a result of evolution in a Newtonian potential are overcome with the application of the inverse scattering technique (IST) [9] to the interface problem [10].

Nevertheless, most nonlinear phenomena arising at nonlinear interfaces have an angular component which is largely removed in any NLS-based analysis due to the assumed paraxial approximation. This limitation is overcome using a Helmholtz nonparaxial framework [11,12], where a nonlinear Helmholtz (NLH) equation [13,14] addresses the full evolution of a broad beam (when compared to the wavelength) propagating at arbitrary angles in relation to the longitudinal

\footnotetext{
*julsan@tel.uva.es
}

axis. Nontrivial Helmholtz corrections have been found in not only bright Kerr, but also dark Kerr [15], two-component [16], boundary [17], bistable [18], and algebraic [19] Helmholtz solitons.

The inherent nonparaxial character of solitons evolving at planar interfaces is captured in a unified, and generalized, Snell's law [20] which addresses the evolution of bright [21] and dark solitons [22,23] impinging on either focusing or defocusing Kerr interfaces, respectively. Besides this off-axis nonparaxiality, on-axis nonparaxiality can also be analyzed in terms of a scalar wave equation provided dramatic reductions in soliton width are avoided, i.e., scenarios of strong focusing are removed from the analysis. Otherwise, the propagation of narrow or ultranarrow beams, not necessarily at large angles, would demand a completely different treatment retaining the vector nature of the fields [24,25]. The nonintegrability of the $\mathrm{NLH}$ equation [26] limits the availability of exact analytical tools for the analysis of nonlinear propagation problems. Nevertheless, working out the direct relation between the NLS equation and on-axis propagation of broad beams under our angular Helmholtz model permits the extraction of valuable predictions applicable to the Helmholtz problem [12] even from the IST results.

In contrast to the large variety of works devoted to bright soliton splitting, no previous work (to our knowledge) has been reported for Kerr defocusing interfaces. Initial-value problems, already proposed to address bright soliton breakup [10], can also play a similar role in the case of dark solitons. Studied in the context of both the NLS equation $[27,28]$ and the general framework of the NLH equation [15], certain initial-value problems are the origin of multisoliton dark patterns composed of a fundamental black soliton and pairs of gray solitons traveling with nonzero transverse velocities. Similar patterns have been recently reported at the planar boundary separating two dissimilar Kerr defocusing media within the context of the Helmholtz theory [23]. Under this framework, a general law governing black and gray soliton refraction has been proposed, although the full characterization of the dark soliton pattern resulting from the interface interaction is still to be unveiled. 
This paper thus presents an analysis of soliton splitting occurring at the interface separating two dissimilar Kerr focusing and defocusing media in the framework of the Helmholtz theory. We devote Sec. II to a brief review of the main results obtained in the Helmholtz analysis of nonlinear interfaces. Section III studies bright soliton splitting organized in two subsections, which correspond to the analysis performed for large and small angles of incidence, respectively. Our aim is to highlight the essential differences found in the breakup process when the full angular content of the problem is preserved, thus overcoming the angular restrictions embedded in previous paraxial analyses. Section IV is devoted to studying soliton splitting at defocusing Kerr interfaces where dark soliton patterns are generated. The main properties of both the resulting primary black soliton and the pairs of gray solitons traveling with nonzero transverse velocities are revealed and contrasted with numerical results. Due to the fundamental role played by numerical simulations in this work, Sec. V details some aspects of the parallel implementation of the numerical methods employed. Finally, Sec. VI summarizes the main conclusions of the paper.

\section{HELMHOLTZ ANALYSIS OF NONLINEAR INTERFACES}

The scheme used in the Helmholtz analysis of nonlinear interfaces is illustrated in Figs. 1(a) and 1(b) for the cases of bright and dark solitons, respectively. The white dotted line represents the planar boundary separating two focusing (defocusing) Kerr media with total refractive index $n_{i}=n_{0 i} \pm$ $\alpha_{i} I, i=1$ and 2 , respectively. The subscript denotes medium $i, n_{0 i}$ and $\alpha_{i}$ are the linear and nonlinear refractive indices of the medium, $I$ is the optical intensity, and \pm accounts for focusing (+) or defocusing (-) media. Assuming that medium 2 has a larger nonlinearity than medium 1 , a fundamental soliton propagating in medium 1 undergoes soliton splitting after entering medium 2. The numerical simulations shown in Figs. 1(a) and 1(b) are obtained from the full numerical integration of the NLH equation

$$
\begin{aligned}
& \kappa \frac{\partial^{2} u}{\partial \zeta^{2}}+j \frac{\partial u}{\partial \zeta}+\frac{1}{2} \frac{\partial^{2} u}{\partial \xi^{2}} \pm|u|^{2} u \\
& \quad=\left[\frac{\Delta}{4 \kappa} \pm(1-\alpha)|u|^{2}\right] \chi(\xi, \zeta) u,
\end{aligned}
$$

(a)

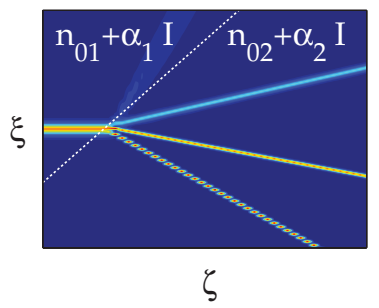

(b)

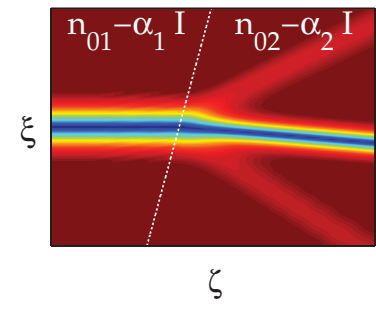

FIG. 1. (Color online) Basic schemes used in this work for Kerr focusing (a) and defocusing (b) interfaces. which describes, for a TE optical field, the evolution of the complex envelope $u$ of a forward-propagating beam. A derivation from the Helmholtz equation is detailed in Ref. [23]. $\chi(\xi, \zeta)$ accounts for the planar boundary which separates the two media, so it takes values 0 or 1 when $(\xi, \zeta)$ is in medium 1 or medium 2 , respectively. In the particular case that the boundary is situated at $\xi=0$, one obtains the Heaviside function $\chi(\xi, \zeta)=H(\xi)$. The normalized transverse and longitudinal coordinates are $\xi=2^{1 / 2} x / w_{0}$ and $\zeta=z / L_{D}$, respectively, and $w_{0}$ is the waist of a reference Gaussian beam with diffraction length $L_{D}=k w_{0}^{2} / 2 . \kappa=$ $1 / k^{2} w_{0}^{2}$ is a nonparaxiality parameter, while $\Delta=1-n_{02}^{2} / n_{01}^{2}$ and $\alpha=\alpha_{2} / \alpha_{1}$ account for the linear and nonlinear refractive index mismatch at the interface, respectively. Bright and dark Helmholtz solitons evolving in medium 1 have been fully described in Refs. [11,12] and Ref. [15], respectively. Exact solutions for the solitons traveling in medium 2 have been reported for bright solitons [20]:

$$
\begin{aligned}
u(\xi, \zeta)= & \eta_{0} \operatorname{sech}\left[\eta_{0} \sqrt{\alpha} \frac{\xi+V \zeta}{\sqrt{1+2 \kappa V^{2}}}\right] \\
& \times \exp \left[j \sqrt{\frac{1-\Delta+2 \kappa \eta_{0}^{2} \alpha}{1+2 \kappa V^{2}}}\left(-V \xi+\frac{\zeta}{2 \kappa}\right)\right] \\
& \times \exp \left(\frac{-i \zeta}{2 \kappa}\right),
\end{aligned}
$$

where $\eta_{0}$ represents the soliton amplitude in medium 1 .

For defocusing Kerr media, a soliton in medium 2 is governed by Ref. [22]

$$
\begin{aligned}
u(\xi, \zeta)= & u_{0}(A \tanh \Theta+j F) \exp \left(\frac{-j \zeta}{2 \kappa}\right) \\
& \times \exp \left[j \sqrt{\frac{1-\Delta-4 \kappa u_{0}^{2} \alpha}{1+2 \kappa V^{2}}}\left(-V \xi+\frac{\zeta}{2 \kappa}\right)\right]
\end{aligned}
$$

where

$$
\Theta=\frac{u_{0} A \alpha^{1 / 2}(\xi+W \zeta)}{\sqrt{1+2 \kappa W^{2}}} \quad \text { and } \quad W=\frac{V-V_{0}}{1+2 \kappa V V_{0}} .
$$

$V$ is a transverse velocity arising from the arbitrary rotation angle $\theta$ of the laboratory coordinates, $V=\tan (\theta)(2 \kappa)^{-1 / 2}$ [11], and $W$ is the net transverse velocity of the dark soliton resulting from the combination of $V$ and the intrinsic transverse velocity $V_{0}=u_{0} F \alpha^{1 / 2}\left[1-\Delta-\left(2+F^{2}\right) 2 \kappa u_{0}^{2} \alpha\right]^{-1 / 2}$. $F=\left(1-A^{2}\right)^{1 / 2}$ is the soliton grayness-contrast parameter and $u_{0}$ is the background amplitude. Of course, in the absence of an interface, $\Delta=0$ and $\alpha=1$, one recovers from Eqs. (2) and (3) the expressions for Helmholtz bright [11] and dark [15] solitons in medium 1.

The angular relationship between solitons propagating in media 1 and 2 is summarized in a compact and generalized Snell's law [23]

$$
\gamma_{ \pm} n_{01} \cos \left(\theta_{n i}+\theta_{0 i}\right)=n_{02} \cos \left(\theta_{n t}+\theta_{0 t}\right)
$$

where $\theta_{n i}$ and $\theta_{n t}$ represent the net angles of incidence and refraction, respectively. For the case of dark solitons, Eq. (5) has to be supplemented with the continuity of the contrast parameter across the interface [22]. The intrinsic angular 
components of an incident and a refracted gray soliton are $\theta_{0 i}$ and $\theta_{0 t}$, respectively, so that one has $\theta_{0 i}=\theta_{0 t}=0$ for the case of bright and black $(F=0)$ solitons [23]. $\gamma_{ \pm}$is a nonlinear correction term which assumes different values for bright $\gamma_{+}[20]$ and dark $\gamma_{-}$[22] solitons.

\section{BRIGHT SOLITON BREAKUP}

\section{A. Nonlinear-step interfaces}

Our analysis of bright soliton splitting will be restricted to nonlinear-step interfaces, i.e., interfaces where linear refractive indices in both media are the same and thus $\Delta=0$. Under this condition, the amount of reflected power at such interfaces is reduced in relation to $\Delta \neq 0$ interfaces. Otherwise, these reflections could affect the number of solitons appearing in medium 2. Second, we consider nonlinear-step interfaces with $\alpha>1$ which is a sufficient condition for the existence of solitons in medium 2 and establishes the necessary condition for the splitting of the solitons when crossing the interface. Therefore, our work is almost restricted to mild on-axis nonparaxiality where any dramatic reduction in soliton width resulting from soliton breakup is avoided in order to maintain the validity of a scalar-based analysis of the problem. Scenarios of very strong focusing will be removed from our study provided the Kerr nonlinearity in the second medium verifies $2 \kappa \eta^{2} \alpha \ll 1$.

In contrast to this, the paraxial analysis performed in Ref. [10] assumes $\Delta \neq 0$. Such interfaces are prone to induce significant reflection losses at the interface or, even worse, inhibit soliton formation in medim 2 as a result of total internal reflection. Working strictly with vanishingly small angles of incidence, these effects become so relevant that they may even mask the expected breakup process in medium 2 . This explains the divergence found between theory and numerics and the absence of numerical data for vanishingly small angles of incidence [10].

\section{B. Preservation of the power flow}

Our theoretical predictions are based on the power flow of a Helmholtz soliton [11]. The preservation of this magnitude at both sides of a nonlinear-step interface can be expressed as

$$
2 \eta_{0} \sqrt{1+2 \kappa \eta_{0}^{2}}=\sum_{k=1}^{N} 2 \eta_{0 k} \sqrt{1 / \alpha+2 \kappa \eta_{0 k}^{2}}
$$

The left side of Eq. (6) is the power flow associated with a soliton of amplitude $\eta_{0}$ propagating in medium 1 . On the right-hand side, we have calculated the power flow contributed by $N$ solitons of amplitudes $\eta_{0 k}$, arising in the breakup process. Since the power flow is conserved [11], this sum can be easily evaluated from Eq. (2) with $\Delta=0$ assuming a distance to the interface that assures a sufficient separation of the emerging soliton. Taking into account that the power flow is independent of the soliton angle of propagation, Eq. (6) is valid for all angles of incidence.

Assuming one works with a Helmholtz type of nonparaxiality where $2 \alpha \kappa \eta_{0}^{2} \ll 1$ still holds, Eq. (6) can be approximated

by

$$
\eta_{0} \approx \frac{1}{\sqrt{\alpha}} \sum_{k=1}^{N} \eta_{0 k}
$$

However, two further simplifications have been assumed in Eq. (6). First, we have supposed that there are no reflections at the interface and all incoming power flow passes through the interface to the second medium. Second, we have considered that the power flow is totally delivered into the newly generated beams and no radiation modes appear. Throughout this work, we will review the conditions where these assumptions are no longer valid.

An additional relationship to be verified by the solitons in medium 2 is deduced from Eq. (2) and establishes that each new soliton must accommodate its shape to the new propagation conditions, so that a scale factor $\alpha^{1 / 2}$ must relate soliton amplitude and width.

\section{Numerical results}

Besides these analytical predictions, massive numerical analyses have been performed. In our simulations, a fundamental bright soliton impinges on different nonlinear-step interfaces at distinct angles of incidence, as illustrated in Fig. 2. The four images show that the number of solitons in medium 2 actually depends on the angle of incidence, which is a remarkable difference from the paraxial predictions [10].

Results for $\theta_{i}=1^{\circ}$ (upper row) and $\theta_{i}=80^{\circ}$ (bottom row) and interfaces with $\alpha=9$ (left column) and $\alpha=6$ (right column) are combined in Fig. 2. Solitons impinging on an interface with $\alpha=9$ at $\theta_{i}=1^{\circ}$ decompose into four solitonlike beams, while only three are obtained at $\theta_{i}=80^{\circ}$. The same difference is observed in the comparison for the case of $\alpha=6$. Nonparaxial scenarios become more restrictive in the number of solitons in medium 2 [29]. Not only the number of solitons but also the amount of reflected power at the interface or the angle of refraction of the largest amplitude beam are so

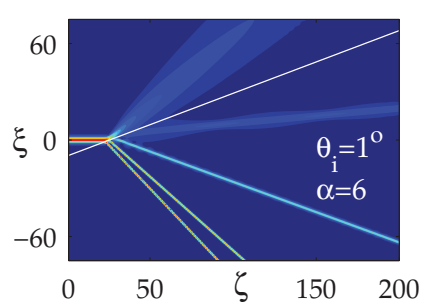

(a)
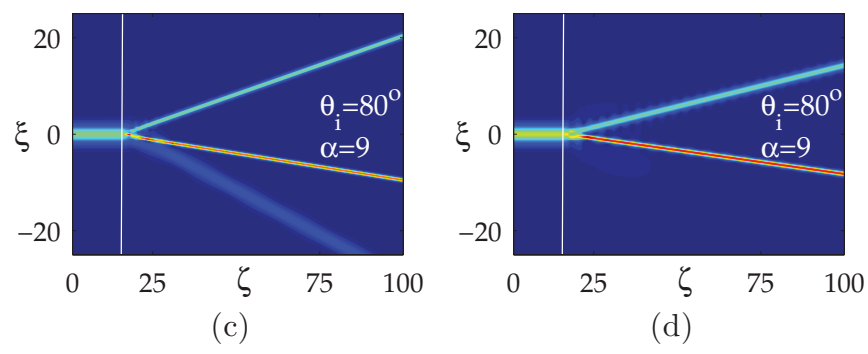

FIG. 2. (Color online) Soliton breakup for $\theta_{i}=1^{\circ}$ (upper row) and $\theta_{i}=80^{\circ}$ (bottom row), while the same values of $\alpha$ are used in each column. In all cases, $\eta_{0}=1$ and $\kappa=10^{-3}$. 
different that we can distinguish between scenarios associated with large and small angles of incidence.

\section{Large angles of incidence}

Our study for large angles of incidence will be illustrated for the reference value of $\theta_{i}=80^{\circ}$ when eight different nonlinearstep interfaces are considered. Table I collects the soliton peak amplitudes $\eta_{0 k}$ and widths $\xi_{0 k}^{-1}$ of the new solitonlike beams generated in medium 2 when the strength of the nonlinearity is increased from a factor of 2 to a factor 9 (first row). These numerical values are used to compute the soliton amplitudewidth ratio $\xi_{0 k}^{-1} / \eta_{0 k}$ and the sum of all numerical amplitudes $\sum \eta_{0 k}$.

As expected, the number of solitonlike beams in the second medium depends on $\alpha$, following a discrete emission process where each new solitonlike beam is formed once a threshold energy level value is reached [10]. Second, the numerical results of Table I confirm that each soliton resulting from soliton breakup accommodates its amplitude-width relationship $\xi_{0 k}^{-1} / \eta_{0 k}$ to the strength of the nonlinear refractive index $\alpha^{1 / 2}$, as predicted by Eq. (2). Finally, the power flow preservation shown in Eq. (7) is also verified, as $\sum \eta_{0 k}$ tends to $\alpha^{1 / 2}$.

The tiny differences found between $\sum \eta_{0 k}$ and $\alpha^{1 / 2}$ can be associated with radiation modes arising in the emission of multiple solitons. This magnitude has been numerically computed, comparing the numerical solution given by the NLH equation and the one composed of $N$ sech-type solitons of amplitude $\eta_{0 k}$. Taking into account that the reflected power flow is negligible for large angles of incidence, as will be discussed in Sec. IIIE, the power radiated in the second medium relative to the incident power is plotted with points in Fig. 3(a) for $\theta_{i}=70^{\circ}$ and $\theta_{i}=80^{\circ}$. The black solid line represents the analytical expression for the normalized radiated power found in previous paraxial studies [10], $P_{\text {rad }}(\%) \sim$ $(1-N / \sqrt{\alpha})^{2}$, where $N$ is the number of solitons formed in the second medium.

The maxima in the radiation pattern are the preludes of new soliton release and are found very close to the threshold values of $\alpha$ which give rise to new solitons. These are found for $\alpha^{1 / 2}=1.5$ and $\alpha^{1 / 2}=2.5$ [10]. The newly generated solitons evolve toward their corresponding stationary states through

TABLE I. Soliton amplitude and width extracted from the numerical simulations when $\theta_{i}=80^{\circ}, \eta_{0}=1$, and $\kappa=10^{-3}$.

\begin{tabular}{lcccccccc}
\hline \hline$\alpha$ & 2 & 3 & 4 & 5 & 6 & 7 & 8 & 9 \\
$\sqrt{\alpha}$ & 1.414 & 1.732 & 2 & 2.373 & 2.449 & 2.646 & 2.828 & 3 \\
$\eta_{01}$ & 1.283 & 1.410 & 1.486 & 1.537 & 1.575 & 1.608 & 1.630 & 1.643 \\
$\eta_{02}$ & & 0.258 & 0.495 & 0.650 & 0.762 & 0.827 & 0.897 & 0.981 \\
$\eta_{03}$ & & & & & & 0.064 & 0.227 & 0.303 \\
$\sum \eta_{0 k}$ & 1.283 & 1.668 & 1.981 & 2.187 & 2.337 & 2.499 & 2.754 & 2.927 \\
$\xi_{01}^{-1}$ & 1.682 & 2.401 & 2.972 & 3.413 & 3.755 & 4.136 & 4.599 & 4.917 \\
$\xi_{02}^{-1}$ & & 0.451 & 0.986 & 1.436 & 1.802 & 2.129 & 2.509 & 2.936 \\
$\xi_{03}^{-1}$ & & & & & & 0.126 & 0.631 & 0.931 \\
$\xi_{01}^{-1} / \eta_{01}$ & 1.311 & 1.703 & 2.000 & 2.221 & 2.384 & 2.572 & 2.821 & 2.993 \\
$\xi_{02}^{-1} / \eta_{02}$ & & 1.748 & 1.99 & 2.210 & 2.496 & 2.574 & 2.797 & 2.991 \\
$\xi_{03}^{-1} / \eta_{03}$ & & & & & & 1.968 & 2.779 & 3.077 \\
\hline \hline
\end{tabular}

(a)
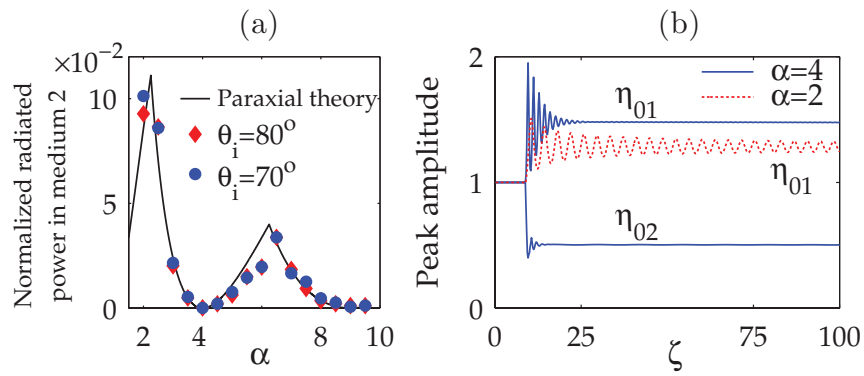

FIG. 3. (Color online) (a) Power radiated in the second medium relative to the incident power for $\theta_{i}=70^{\circ}$ (circles) and $\theta_{i}=80^{\circ}$ (diamonds). (b) Soliton peak amplitude for $\theta_{i}=80^{\circ}$ when $\alpha=4$ (solid blue) and $\alpha=2$ (dotted red). In all cases, $\eta_{0}=1$ and $\kappa=10^{-3}$.

a series of long-lived amplitude oscillations where radiation is gradually released. This is shown in Fig. 3(b) for the case of $\alpha=2$ which illustrates the evolution of the soliton peak amplitude along the longitudinal coordinate. On the other hand, the minima found for $\alpha^{1 / 2} \in \mathrm{Z}$ in Fig. 3(a) reveal that the power flow supplied to the medium fits perfectly into the discrete emission pattern associated with the energy levels of the new medium in the large-angle regime, so that solitons propagate with negligible changes in shape once they have crossed the nonlinear interface. Such behavior is captured in Fig. 3(b) when one observes the amplitude evolution of the two solitons generated for the case of $\alpha=4$.

Figure 3 also reveals that the way radiation is released for very large angles of incidence fits well the analytical predictions of previous paraxial studies. Such agreement is also found when one computes the threshold values of $\alpha$ which originate an additional soliton, and confirmed when one compares the soliton peak amplitudes of Table I with the relationships provided in Ref. [10]. In contrast to other interface-related phenomena, very good agreement between Helmholtz theory and paraxial descriptions is found here at large angles of incidence, since this condition defines a regime very close to normal incidence where the perturbation induced by the interface can be dealt with using the predictions of the paraxial theory [12].

\section{E. Small angles of incidence}

Leaving aside the differences found in the number of solitons generated at the interface, two additional features are revealed in the plots of Fig. 2 when small and large angles of incidence are compared. First, the reflections increase significantly at low angles of incidence. In addition to this, the interaction between the soliton beam and the interface spreads over a longer distance, so that the perturbation effects introduced by the interface have a deeper impact on soliton decomposition. Both effects make the predictions of the paraxial theory diverge from our numerical results collected in Table II, which represent for $\theta_{i}=1^{\circ}$ the same magnitudes as in Table I. Neither the number of solitons nor their amplitudes correspond to the predictions of the paraxial theory. Only the ratios $\xi_{0 k}^{-1} / \eta_{0 k}$ tend to $\alpha^{1 / 2}$, showing that the solitons accommodate their amplitude-width relationship to the predictions of Eq. (2). 
TABLE II. Soliton amplitude and width extracted from the numerical simulations when $\theta_{i}=1^{\circ}, \eta_{0}=1$, and $\kappa=10^{-3}$.

\begin{tabular}{lcccccccc}
\hline \hline$\alpha$ & 2 & 3 & 4 & 5 & 6 & 7 & 8 & 9 \\
$\sqrt{\alpha}$ & 1.414 & 1.732 & 2 & 2.373 & 2.449 & 2.646 & 2.828 & 3 \\
$\eta_{01}$ & 1.211 & 1.218 & 1.178 & 1.128 & 1.079 & 1.033 & 0.992 & 0.954 \\
$\eta_{02}$ & & 0.302 & 0.518 & 0.639 & 0.713 & 0.760 & 0.787 & 0.802 \\
$\eta_{03}$ & & & & & 0.221 & 0.318 & 0.392 & 0.453 \\
$\eta_{04}$ & & & & & & & & 0.145 \\
$\sum_{0 k}^{-1} \eta_{01}$ & 1.211 & 1.520 & 1.696 & 1.767 & 2.013 & 2.111 & 2.171 & 2.354 \\
$\xi_{01}^{-1}$ & 1.687 & 2.089 & 2.334 & 2.509 & 2.633 & 2.725 & 2.797 & 2.855 \\
$\xi_{02}^{-1}$ & & 0.512 & 1.006 & 1.214 & 1.731 & 1.996 & 2.216 & 2.399 \\
$\xi_{03}^{-1}$ & & & & & 0.456 & 0.797 & 1.046 & 1.307 \\
$\xi_{04}^{-1}$ & & & & & & & & 0.445 \\
$\xi_{01}^{-1} / \eta_{01}$ & 1.392 & 1.715 & 1.981 & 2.224 & 2.440 & 2.638 & 2.820 & 2.992 \\
$\xi_{02}^{-1} / \eta_{02}$ & & 1.695 & 1.942 & 1.900 & 2.428 & 2.626 & 2.816 & 2.991 \\
$\xi_{03}^{-1} / \eta_{03}$ & & & & & 2.063 & 2.506 & 2.669 & 2.886 \\
$\xi_{04}^{-1} / \eta_{04}$ & & & & & & & & 3.069 \\
\hline \hline
\end{tabular}

Our numerical analysis evaluates first the reflected power flow generated at the interface for different values of $\alpha$ and angles of incidence. The ratio between the reflected and incoming power flow determines a reflection coefficient which is shown in Fig. 4. Each point has been numerically obtained based on the power flow evolution along the longitudinal coordinate $\zeta$, as the inset of Fig. 4 illustrates. We define the power flow via the interface as the amount of incoming power that is transmitted into medium 2, so that the power flow evaluated for each medium far away from the interface (for instance, at $\zeta_{0}=150$ ) gives the amount of reflected and transmitted power flow. Figure 4 shows that larger values of $\alpha$ make the reflection coefficient increase. Moreover, the reflected power flow is significant only for small angles of incidence, thus explaining why we omitted this effect previously in the analysis of large angles of incidence.

Based on the results of Fig. 4, we can define for each value of $\alpha$ and $\theta_{i}$ a transmission coefficient $t_{\alpha, \theta}=1-r_{\alpha, \theta}$ which

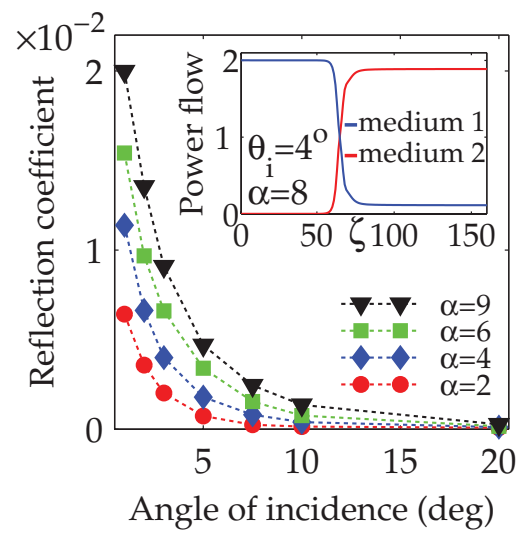

FIG. 4. (Color online) Reflection coefficient as a function of the angle of incidence for different nonlinear interfaces.
TABLE III. Transmission coefficient for very small angles of incidence. In all cases, $\eta_{0}=1$ and $\kappa=10^{-3}$.

\begin{tabular}{ccccccc}
\hline \hline$\alpha$ & $t_{\alpha, 1^{\circ}}$ & $\sum \eta_{0 k} / \sqrt{\alpha}$ & $t_{\alpha, 2^{\circ}}$ & $\sum \eta_{0 k} / \sqrt{\alpha}$ & $t_{\alpha, 3^{\circ}}$ & $\sum \eta_{0 k} / \sqrt{\alpha}$ \\
\hline 2 & 0.9358 & 0.8563 & 0.9642 & 0.8664 & 0.9798 & 0.8756 \\
3 & 0.9170 & 0.8776 & 0.9544 & 0.9299 & 0.9722 & 0.9493 \\
4 & 0.8861 & 0.8480 & 0.9337 & 0.9136 & 0.9600 & 0.9470 \\
5 & 0.8641 & 0.7902 & 0.9133 & 0.8664 & 0.9432 & 0.9091 \\
6 & 0.8458 & 0.8212 & 0.9032 & 0.8752 & 0.9339 & 0.8902 \\
7 & 0.8272 & 0.7979 & 0.8921 & 0.8666 & 0.9289 & 0.9249 \\
8 & 0.8130 & 0.7676 & 0.8793 & 0.8510 & 0.9191 & 0.9026 \\
9 & 0.8002 & 0.7847 & 0.8645 & 0.8276 & 0.9091 & 0.8878 \\
\hline \hline
\end{tabular}

gives the amount of power flow transmitted to the second medium. Taking this into account, Eq. (7) must be corrected:

$$
\eta_{0} t_{\alpha, \theta} \sim \frac{1}{\sqrt{\alpha}} \sum_{i=k}^{N} \eta_{0 k},
$$

so that the transmitted power flow injected into the second medium is now scaled by $t_{\alpha, \theta}$. Table III collects the numerical values of both terms involved in Eq. (8) for $\theta_{i}=1^{\circ}, 2^{\circ}$, and $3^{\circ}$, and the same values of $\alpha$ used in Tables I and II.

In all cases, one obtains $t_{\alpha, \theta}>\alpha^{-1 / 2} \sum \eta_{0 k}$, so that the tiny differences between the two terms are the result of the power radiated by the newly generated solitons in medium 2 . This magnitude relative to the incident power has been numerically calculated as in Fig. 4 and plotted in Fig. 5. Diamonds, circles, and squares represent numerical values while the solid black line represent the same theoretical prediction of the paraxial theory which was plotted in Fig. 3(a).

Unlike the results of Fig. 3, radiation patterns for small angles of incidence do not match the analytical predictions of the paraxial theory (solid black). The NLS-based description of soliton breakup [10] thus fails when small angles of incidence come into play. This situation is similar to initial-value problems which exhibit an angular character, so that they cannot be properly addressed within the restrictions of paraxial analyses [12].

The three numerical curves in Fig. 5 also suggest that the radiation pattern moves to the right side as the angle of incidence increases. The numerical results for angles of

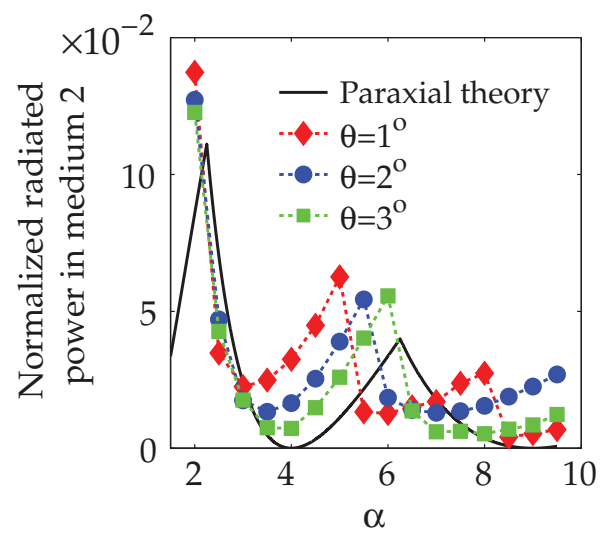

FIG. 5. (Color online) Power radiated in the second medium relative to the incident power at $\theta_{i}=1^{\circ}$. 
incidence in the vicinity of $\theta_{i}=5^{\circ}$ are quite similar to those shown in Fig. 3(a), thus establising the angular threshold delimiting what we have defined as the small and large angular regimes, respectively. If we now focus on the results for $\theta_{i}=1^{\circ}$, the three peaks located at $\alpha=2,5$, and 8 reveal that an additional soliton is found in the vicinity of $\alpha=2.5,5.5$, and 8.5, respectively. That explains why we chose $\alpha=6$ and 9 in Fig. 2 to highlight the angular dependency on the number of solitons.

\section{F. Angle of refraction}

The angle of incidence may affect not only the number of solitons appearing in the second medium, but also the way the new multisoliton pattern arises. This is shown in Fig. 6 which illustrates the evolution of a Helmholtz soliton entering the same nonlinear medium $(\alpha=4)$ with different angles of incidence, namely, $\theta_{i}=1^{\circ}, 20^{\circ}$, and $80^{\circ}$ corresponding to Figs. 6(a), 6(b), and 6(c), respectively. For large enough angles of incidence, the divergence among the emerging beams decreases, as shown in Fig. 6(c) where the two solitons appear as single entities far away from the interface. This behavior has already been observed in previous paraxial work [10].

Our Helmholtz analysis, however, goes further: the angle of refraction of the largest-amplitude beam in medium 2 fits the predictions of the generalized Snell's law. Nonlinearstep interfaces with $\alpha>1$ lead to external refraction, as is represented by the set of lines in Fig. 6(d) obtained for $\alpha=2$ (dotted black), $\alpha=5$ (solid red), and $\alpha=9$ (dashed blue). The numerical results, represented by the series of circles, squares, and diamonds, agree with theoretical predictions showing that the angle of refraction tends to the angle of incidence as this increases. This behavior is captured in the plots of Fig. 6 where the angle of refraction of the largest-amplitude soliton in medium 2 is displayed. (a)
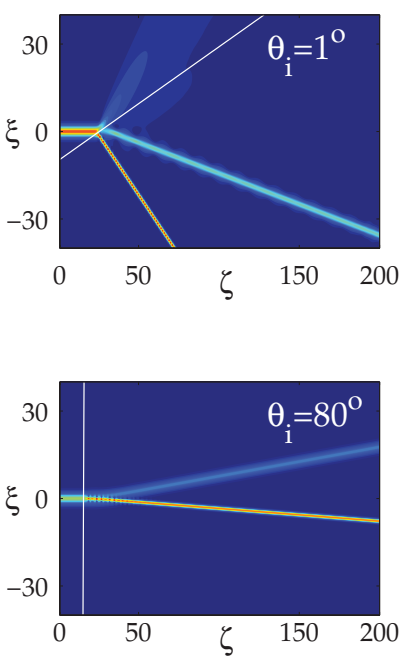

(c) (b)
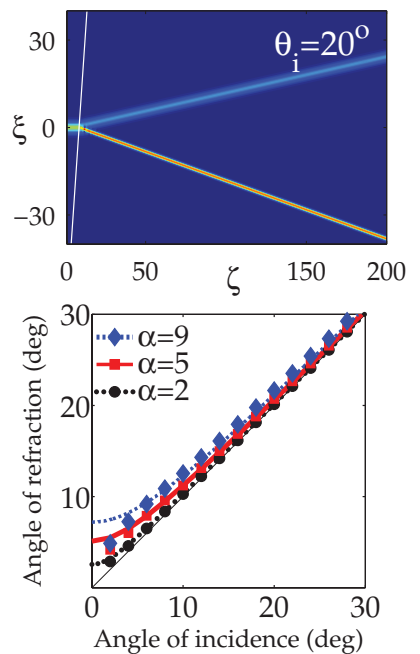

(d)
FIG. 6. (Color online) Soliton decomposition for $\alpha=4$ and different angles of incidence $\theta_{i}=1^{\circ}(\mathrm{a}), \theta_{i}=20^{\circ}(\mathrm{b})$, and $\theta_{i}=80^{\circ}$ (c). (d) Angle of refraction for the soliton with the largest amplitude for nonlinear-step interfaces and $\kappa=10^{-3}$.

\section{DARK SOLITON BREAKUP}

We now consider the circumstances and consequences of multiple dark solitons [30] being generated at nonlinear interfaces. Although the refraction of dark solitons has been analyzed within the framework of the Helmholtz theory [22,23], only the angular relationships between single incident and refracted solitons have been studied. The full characterization of multisoliton patterns emerging upon crossing a nonlinear interface is still lacking and constitutes the main object of this section.

In the case of focusing Kerr media, we have considered the role of an interface in terms of two, potentially distinct, effects, i.e., reflection at the interface and radiation generation. The former has been found to play a significant role for small angles of incidence, being negligible for large angles of incidence. Such an angular clasification of the problem fails completely for the case of defocusing Kerr interfaces. Dark soliton splitting can be analyzed only for large angles of incidence, so that the background beam supporting the soliton dip is not severely affected by the perturbation that the interface represents.

\section{A. Black soliton breakup}

We address here the splitting of black solitons upon traversing a nonlinear interface. Pairs of gray solitons propagating at opposite angles can be generated in a Kerr medium whenever the initial condition does not correspond to the fundamental black soliton. Within the paraxial approximation, an initial condition of the form $u(\xi, 0)=u_{0} \tanh (a \xi)$ leads to the generation of a set of gray solitons whose number, amplitude, and transverse velocity depend on the ratio $u_{0} / a[27,28]$. Nontrivial corrections in soliton properties have been reported for the same type of problem in the general nonparaxial description [15].

Our interface problem here can be described in a similar way provided $a$ is replaced by $\alpha^{-1 / 2}$. A fundamental black soliton in medium 1 which impinges on medium 2 with a larger Kerr nonlinearity $\alpha>1$ can create, besides a black soliton, $2 N_{0}$ additional gray solitons, where $N_{0}$ is the largest integer satisfying $N_{0}<\sqrt{\alpha}$. Thus, the slightest increase in nonlinearity across the interface will give rise to an additional pair of gray solitons. This feature is consistent with the thresholdlesslike character of gray soliton generation [28], and is in contrast with the thresholds encountered for generating additional bright solitons at interfaces separating two focusing Kerr media.

Figures 7(a) and 7(b) illustrate the creation of two and four additional solitons when $\alpha=4$ and $\alpha=9$. Also, for each simulation, $\alpha$ was chosen to be immediately below the threshold for generation of a further two gray solitons and, again, such additional solitons are not observed in either case.

Both plots of Fig. 7 also illustrate the investigation carried out on black soliton breakup. A fundamental black soliton is launched at distinct angles of incidence on different nonlinear interfaces which, unlike the case of bright breakup, are not restricted to nonlinear-step interfaces. In fact, we have assumed $\Delta<0$ in Fig. 7 to work under the total transparency condition for black solitons [22], thus assuring that the refracted 
(a)

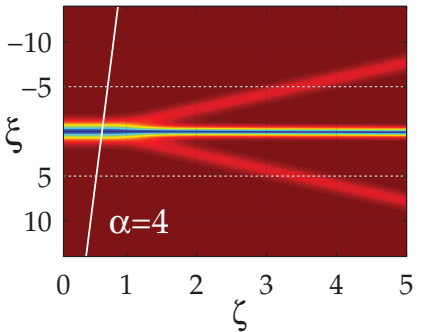

(b)

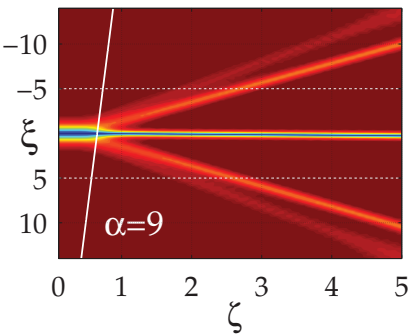

FIG. 7. (Color online) (a) Two additional gray solitons are generated when $\Delta=-1.5 \times 10^{-2}, \alpha=4$, and $\theta_{n i}=70^{\circ}$. (b) Four new gray solitons appear when $\alpha=9, \Delta=-5.4 \times 10^{-2}$ and $\theta_{n i}=$ $80^{\circ}$. In both cases, $u_{0}=1$ and $\kappa=10^{-3}$.

fundamental dark soliton preserves the angle of incidence. However, we find that for large values of $\alpha$ this condition only holds approximately. If we also exploit the rotational symmetry that the Helmholtz framewok provides [11,23], the refracted black soliton will propagate in medium 2 along the longitudinal coordinate, so that the gray soliton pairs arising at the interface will exhibit a symmetric pattern.

\section{B. Fundamental black soliton}

We study first the main features of the refracted fundamental black soliton. The direction of propagation of the primary black soliton in medium 2 obeys the generalized Snell's law, as shown in Ref. [23]. However, Figs. 7(a) and 7(b) also show that a pronounced narrowing of the primary soliton is visible for those interfaces with larger $\alpha$. This feature is expected, for the correct soliton shape in strongly nonlinear media, and is already accounted for within the solution matching involved in the derivation of Eq. (3).

Numerical evidence of this result is displayed in Fig. 8, which shows the soliton width evolution along $\zeta$ in relation to the soliton width of a fundamental black soliton which impinges on five different nonlinear interfaces at the reference angle of incidence $\theta_{i}=70^{\circ}$. After an abrupt change occurring close to the interface, the soliton width evolves toward a stationary value in medium 2 . The set of lines in the left side of Fig. 8 correspond to the value of $\alpha^{1 / 2}$ that is the theoretical soliton width in medium 2 predicted by Eq. (3). The agreement

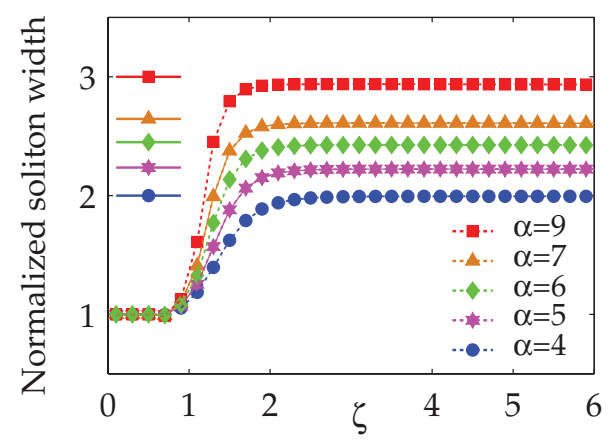

FIG. 8. (Color online) Width evolution of the primary refracted black soliton for different values of $\alpha$.

between numerics and theoretical results holds for most cases. Only for $\alpha=9$ is a tiny mismatch between numerics and analysis found, as a result of the radiation released at the interface on using large values of $\Delta$ to ensure $\delta_{-} \rightarrow 0$. The beam dynamics resulting from the interface interaction in Fig. 8 is similar to that of Ref. [15] arising from initial-value problems. In fact, nonlinear interfaces are shown to provide an excellent scenario for the spontaneous generation of Helmholtz solitons.

\section{Gray soliton pairs}

We now turn our attention to the analysis of the pairs of gray solitons generated at the planar boundary. This problem has been analytically solved for the NLS equation when an initial-value problem of the form $u(0, \xi)=u_{0} \tanh (a \xi)$ is used [28]. The nonparaxial theory for the same type of initial-value problem described before reveals that the transverse velocity of Helmholtz gray soliton pairs evolves according to the nonparaxial predictions [15].

In our interface problem, the transverse velocity of a dark soliton traveling in medium 2 after being refracted by a nonlinear interface is expected to evolve according to Ref. [23]

$$
V_{0}=\frac{u_{0} F \alpha^{1 / 2}}{\left[1-\Delta-\left(2+F^{2}\right) 2 \kappa u_{0}^{2} \alpha\right]^{1 / 2}},
$$

where all parameters have been defined in Eq. (4). Taking into account that we make the refracted primary black soliton propagate in medium 2 along the longitudinal axis, Eq. (9) thus gives the net transverse velocity of the first pair of gray solitons in relation to the refracted primary black soliton, as represented in Fig. 9(a). The angle $\Delta \theta$ associated with $V_{0}$ in the unscaled coordinate system, $\tan ^{2} \theta=2 \kappa V^{2}$ [11], is

$$
\Delta \theta=\tan ^{-1}\left\{\sqrt{\frac{2 \kappa u_{0}^{2} F^{2} \alpha}{1-\Delta-\left(2+F^{2}\right) 2 \kappa u_{0}^{2} \alpha}}\right\} .
$$

In order to test the validity of Eq. (10), we made a fundamental black soliton impinge on different nonlinear interfaces at distinct angles of incidence as shown in Fig. 9(a). Numerical results for $\Delta \theta$ are displayed with large black diamonds $(\alpha=9)$, green triangles $(\alpha=7)$, red squares $(\alpha=$ 5), and blue circles $(\alpha=3)$ in Fig. 9(b), revealing that the angle of refraction of gray soliton pairs (when compared to the angle of the primary black soliton) increases for larger values of $\alpha$ and remains independent of the angle of incidence. (a)

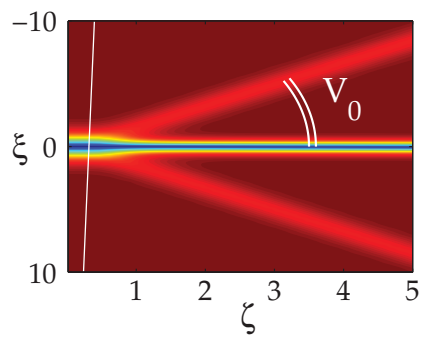

(b)

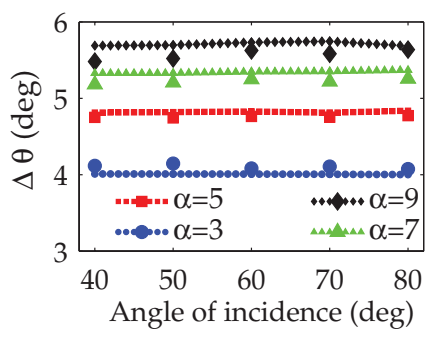

FIG. 9. (Color online) (a) Definition of $V_{0}$. (b) Numerical values (points) and predictions (lines) for $\Delta \theta$ and different values of $\alpha$. 


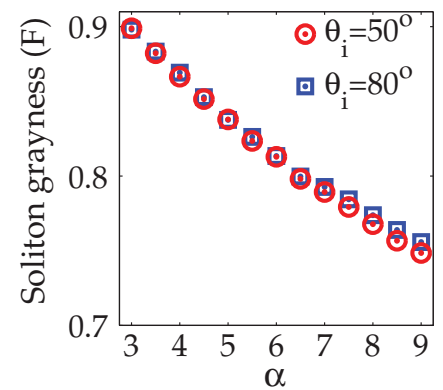

FIG. 10. (Color online) Grayness of gray soliton pairs for different values of $\alpha$ and $\theta_{i}$. In all cases, $u_{0}=1$ and $\kappa=10^{-3}$.

The black diamonds ( $\alpha=9)$, green triangles $(\alpha=7)$, red squares $(\alpha=5)$, and blue circles $(\alpha=3)$ represent Eq. (10) for the same set of parameters used in the simulations, i.e., $u_{0}=1, \kappa=10^{-3}$, and interface parameters in the vicinity of the total transparency condition. The value of $F$ in Eq. (10) has been extracted from simulations and is shown in Fig. 10 for distinct nonlinear interfaces and two angles of incidence. The larger the nonlinear mismatch, the darker is the gray soliton pair. This can also be observed in the two images of Fig. 7 for $\alpha=4$ and $\alpha=9$.

The analysis performed here has focused on the primary gray soliton pairs associated with the lower terms $(n=1)$ of the initial-value problem [27]. Additional pairs obtained for $n>1$ will reduce their darkness and, hence, undergo a larger divergence. This can be seen in Fig. 7(b) where two additional pairs behave according to these predictions.

\section{Asymmetries in the decomposition}

We also find another interface-related feature present for both black and gray soliton refraction, which is that multiplesoliton generation exhibits asymmetries in the directions and widths of the resultant beams when the total transparency restriction is removed from the analysis.

Figures 11(a) and 11(b) illustrate this effect for external $\left(\delta_{-}<0\right)$ and internal $\left(\delta_{-}>0\right)$ refraction, respectively. Similar results have also been demonstrated for gray soliton refraction for which (to our knowledge) no perturbed gray initial-value problem has been reported. (a)

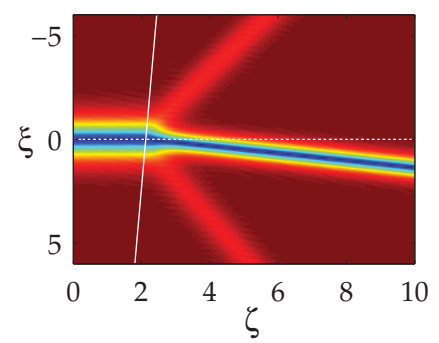

(b)

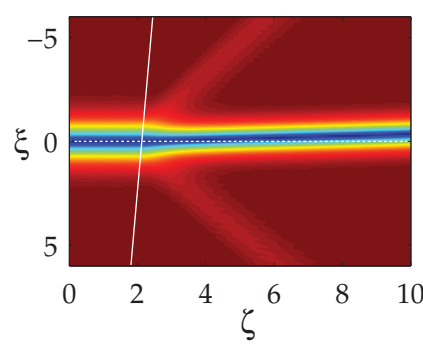

FIG. 11. (Color online) Asymmetrical dark soliton patterns obtained when $\Delta=-2.2 \times 10^{-2}$ and $\alpha=4$ (a), and $\Delta=10^{-3}$ and $\alpha=2.5$ (b). In both cases, $u_{0}=1, \kappa=10^{-3}$, and $\theta_{i}=40^{\circ}$.

\section{Numerical results}

Massive numerical simulations have played an essential role in this paper. Not only bright but also dark soliton splitting has been simulated based on a nonparaxial beam propagation method [31], which has become a powerful tool to contrast the validity of the analytical predictions of the Helmholtz theory. It is a difference-differential method [31] which combines finite differences and spectral methods. The second initial condition is obtained from the exact expression of the incident soliton [11]. Simulations for both focusing and defocusing Kerr media have exploited the rotational symmetry that the Helmholtz framework provides. Instead of launching solitons with a nonzero angle of propagation that impinge on a planar boundary placed at $\xi=0$, the interface is rotated, so that solitons propagating with zero transverse velocity encounter the interface obliquely placed at the same angle.

The main difference between the simulations of bright and dark soliton splitting is the amount of propagation steps needed in each case. While simulations for bright solitons require $2 \times$ $10^{6}$ propagation steps, black soliton simulations demanded only $2 \times 10^{5}$. We have employed in this paper the same finite background beam as in Ref. [23], i.e., a raised cosine: $h(\xi)=\cos ^{2}\left[\pi / r L\left(|\xi|-L_{1}\right)\right]$, if $L_{1}<|\xi|<L_{2} ; h(\xi)=1$, if $|\xi| \leqslant L_{1}$; and $h(\xi)=0$, if $|\xi| \geqslant L_{2}$, where the roll-off factor $r=0.5$, grid length $L=160, L_{1}=(1-r) L / 4$, and $L_{2}=$ $(1+r) L / 4$. We have thus assured that the generated dark soliton pattern at the interface evolves on the flat background the necessary propagation steps to extract the main properties of the supported dark solitons. The transverse coordinate has been sampled with $N=115200=2^{9} \times 15^{2}$ data points to run efficiently on 15 cores or processors of our parallel computing systems [32]. In this work, the symmetric multiprocessor system used is a HP Proliant DL580 G5 composed of four 4 Intel Xeon 7320 Quad core processors running at $2.13 \mathrm{GHz}$.

\section{CONCLUSIONS}

In this work we have analyzed soliton breakup at nonlinear interfaces separating two focusing and defocusing Kerr-type media within the framework of the Helmholtz theory, thus preserving the full angular content of the problem. This has allowed us to demonstrate, in the case of bright solitons, that soliton breakup depends strongly on the angle of incidence. Such angular dependency, absent from a NLS equation-based analysis, is manifest not only in the number of solitons formed in the new medium, but also in the amount of reflected power at the interface or in the radiation modes appearing in the new nonlinear medium. Unlike paraxial analyses, the Helmholtz framework accommodates the angular character that initialvalue problems may exhibit. Our Helmholtz analysis has been compared with previous paraxial works based on the NLS equation, where agreement between paraxial and Helmholtz results was found to be limited only to large angles of incidence.

The same Helmholtz theory has allowed us to analyze the generation of dark soliton patterns at a nonlinear interface separating two defocusing Kerr media. We have fully determined the main properties of the resulting soliton pattern in medium 2 composed of a fundamental black soliton and pairs 
of gray solitons traveling with nonzero transverse velocities. Excellent agreement between Helmholtz-based predictions and numerical results obtained from the numerical integration of the NLH equation has been found throughout this work.

\section{ACKNOWLEDGMENT}

This work was supported by the Spanish Ministerio de Educación y Ciencia and Fondo Europeo de Desarrollo Regional, Project No. TEC2010-21303-C04-04.
[1] N. N. Rozanov, Opt. Spektrosk. 47, 606 (1979) [Opt. Spectrosc. 47, 335 (1979)].

[2] W. J. Tomlinson, J. P. Gordon, P. W. Smith, and A. E. Kaplan, Appl. Opt. 21, 2041 (1982).

[3] E. M. Wright, G. I. Stegeman, C. T. Seaton, J. V. Moloney, and A. D. Boardman, Phys. Rev. A 34, 4442 (1986).

[4] E. M. Wright, D. R. Heatley, and G. I. Stegeman, Phys. Rep. 194, 309 (1990).

[5] F. Ye, Y. V. Kartashov, and L. Torner, Opt. Lett. 32, 394 (2007).

[6] V. A. Aleshkevich, Y. V. Kartashov, A. S. Zelenina, V. A. Vysloukh, J. P. Torres, and L. Torner, Opt. Lett. 29, 483 (2004).

[7] L. A. Nesterov, Opt. Spektrosk. 64, 1166 (1988) [Opt. Spectrosc. 64, 694 (1988)].

[8] A. B. Aceves, J. V. Moloney, and A. C. Newell, Phys. Rev. A 39, 1809 (1989).

[9] V. E. Zakharov and A. B. Shabat, Zh. Eksp. Teor. Fiz 61, 118 (1971) [Sov. Phys. JETP 34, 62 (1972)].

[10] A. B. Aceves, J. V. Moloney, and A. C. Newell, Phys. Rev. A 39, 1828 (1989).

[11] P. Chamorro-Posada, G. S. McDonald, and G. H. New, J. Mod. Opt. 45, 1111 (1998).

[12] P. Chamorro-Posada, G. S. McDonald, and G. H. New, J. Mod. Opt. 47, 1877 (2000).

[13] G. Fibich, Phys. Rev. Lett. 76, 4356 (1996).

[14] P. Chamorro-Posada, G. S. McDonald, and G. New, J. Opt. Soc. Am. B 19, 1216 (2002).

[15] P. Chamorro-Posada and G. S. McDonald, Opt. Lett. 28, 825 (2003).

[16] J. M. Christian, G. S. McDonald, and P. Chamorro-Posada, Phys. Rev. E 74, 066612 (2006).
[17] J. M. Christian, G. S. McDonald, and P. Chamorro-Posada, J. Phys. A 40, 1545 (2007).

[18] J. M. Christian, G. S. McDonald, and P. Chamorro-Posada, Phys. Rev. A 76, 033833 (2007).

[19] J. M. Christian, G. S. McDonald, and P. Chamorro-Posada, J. Phys. A 43, 085212 (2010).

[20] J. Sánchez-Curto, P. Chamorro-Posada, and G. S. McDonald, Opt. Lett. 32, 1126 (2007).

[21] J. Sánchez-Curto, P. Chamorro-Posada, and G. S. McDonald, J. Opt. A: Pure Appl. Opt. 11, 054015 (2009).

[22] J. Sánchez-Curto, P. Chamorro-Posada, and G. S. McDonald, Opt. Lett. 35, 1347 (2010).

[23] J. Sánchez-Curto, P. Chamorro-Posada, and G. S. McDonald, Phys. Rev. A 83, 013828 (2011).

[24] B. Crosignani, P. Di Porto, and A. Yariv, Opt. Lett. 22, 778 (1997).

[25] A. Ciattoni, P. Di Porto, B. Crosignani, and A. Yariv, J. Opt. Soc. Am. B 17, 809 (2000).

[26] P. Chamorro-Posada and G. S. McDonald, Phys. Rev. E 74, 036609 (2006).

[27] W. Zhao and E. Bourkoff, Opt. Lett. 14, 703 (1989).

[28] Yu. S. Kivshar, IEEE J. Quantum Electron. 29, 250 (1993).

[29] P. Chamorro-Posada, J. Sánchez-Curto, J. M. Christian, and G. S. McDonald, in Proceedings of the Second International Conference on Advanced Optoelectronics and Lasers (CAOL 2005), edited by I. A. Sukhoivanov (IEEE Operations Centre, Piscataway, NJ, 2005), pp. 108-112.

[30] Yu. S. Kivshar and B. Luther-Davies, Phys. Rep. 298, 81 (1998).

[31] P. Chamorro-Posada, G. S. McDonald, and G. New, Opt. Commun. 192, 1 (2001).

[32] J. Sánchez-Curto and P. Chamorro-Posada, Parallel Comput. 34, 539 (2008). 